\title{
On the Modified Optimal Investment Strategy for Annuity Contracts under the Constant Elasticity of Variance (CEV) Model
}

\author{
K. N. C. Njoku ${ }^{1}$ and B. O. Osu ${ }^{2}$ \\ ${ }^{1}$ Department of Mathematics, Imo State University, Owerri, Imo State, Nigeria \\ ${ }^{2}$ Department of Mathematics, Michael Okpara University of Agriculture, Umudike, Abia State, Nigeria
}

\begin{abstract}
In this work, the optimal pension wealth investment strategy during the decumulation phase, in a defined contribution (DC) pension scheme is constructed. The pension plan member is allowed to invest in a risk free and a risky asset, under the constant elasticity of variance (CEV) model. The explicit solution of the constant relative risk aversion (CRRA) and constant absolute risk aversion (CARA) utility functions are obtained, using Legendre transform, dual theory, and change of variable methods. It is established herein that the elastic parameter, $\beta$, say, must not necessarily be equal to one $(\beta \neq 1)$. A theorem is constructed and proved on the wealth investment strategy. Observations and significant results are made and obtained, respectively in the comparison of our various utility functions and some previous results in literature.
\end{abstract}

\section{Introduction}

There are two major designs of pension plan, namely, the defined benefit (DB) pension, and the defined contribution (DC) pension plan. As the names implies, in that of the $\mathrm{DB}$, the benefits of the plan member are defined, and the sponsor bears the financial risk. Whereas, in the DC pension plan, the contributions are defined, the retirement benefits depends on the contributions and the investment returns, and the contributors (the plan members) bears the financial risk. Recently, the DC pension has taken

Received: November 20, 2018; Accepted: December 15, 2018

2010 Mathematics Subject Classification: 91B28, 62P20, 91G50.

Keywords and phrases: annuity contracts, CRRA, CARA, DC, CEV, optimal strategy.

Copyright (C) 2019 K. N. C. Njoku and B. O. Osu. This is an open access article distributed under the Creative Commons Attribution License, which permits unrestricted use, distribution, and reproduction in any medium, provided the original work is properly cited. 
dominance over the DB pension plan in the pension scheme, since DC pension plan is fully funded, which makes it easier for the plan managers (Pension Fund Administrators (PFAs')) and the Pension Fund Custodians (PFCs') to invest equitably in the market, and also makes it easier for the plan members to receive their retirement benefit as and when due.

Investment strategies of the contributions, which in turn is a strong determinant of the investment returns vis-a-vis the benefits of the contributors at retirement must be given optimum attention. Recent publications in economic journals and other reputable mathematics and science journals have brought to light, variety of methods of optimizing investment strategies and returns. For instance, some researchers have made various contributions in this direction, particularly, in DC Pension Plan. Cairns et al. [4] did a work on, "stochastic life styling: optimal dynamic asset allocation for defined contribution pension plans. In their work, various properties and characteristics of the optimal asset allocation strategy, both with and without the presence of non-hedge able salary risk were discussed. The significance of alternative optimal strategy by pension providers was established. Wang and Chen [15] investigated a defined contribution (DC) pension plan investment problem during the accumulation phase under the multi-period mean-variance criterion. Mwanakatwe et al. [14] analysed the optimal investment strategies for a DC pension fund under the Hull-White interest rate model. Under this model, the pension fund manager can invest capital in the bank account, stock index, and real estates. More so, Battocchio and Menoncin [2] studied optimal pension management in a stochastic framework, they came out with a significant result.

In order to deal with optimal investment strategy, the need for maximization of the expected utility of the terminal wealth became necessary. Example, the Constant Relative Risk Aversion (CRRA) utility function, and (or) the Constant Absolute Risk Aversion (CARA) utility function were used to maximize the terminal wealth. Cairns et al. [4], Gao [8], Boulier et al. [3], Deelstra et al. [7], and Xiao et al. [16] used CRRA to maximize terminal wealth. However, Gao [10] used the CRRA and the CARA to maximize terminal wealth, and this triggered our research. Ours is a modification of his work, by considering different categories of contributors, with some other additional assumptions made. Our task in this work is to establish, with a theorem the fact that the elastic parameter $\beta \neq 1$, which is lacking in his work. We used a similar approach in obtaining some of our results. 


\subsection{Preliminaries}

We start with a complete and frictionless financial market that is continuously open over the fixed time interval $[0, T]$, for $T>0$, representing the retirement time of any plan member.

We assume that the market is composed of the risk-free asset (cash), and risky asset (stock). Let $(\Omega, F, P)$ be a complete probability space, where $\Omega$ is a real space and $P$ is a probability measure, $\left\{w_{s}(t), w_{t}(t)\right\}$ are two standard unorthogonal Brownian motions, $\left\{F_{t}(t), F_{S}(t)\right\}$ are right continuous filtrations whose information are generated by the two standard Brownian motions $\left\{w_{s}(t), w_{t}(t)\right\}$, whose sources of uncertainties are respectively to the stock market and time evolution.

\section{Methodology}

\subsection{Hamilton-Jacobi-Bellman (HJB) equation}

Assume we represent $u=u_{s}$ as the strategy and we define the utility attained by the contributor from a given state $y$ at time $t$ as

$$
\phi_{u}(t, r, y)=E_{u}[U(Y(t)): r(t)=r, y(t)=y],
$$

where $t$ is the time, $r$ is the short interest rate and $y$ is the wealth. Our interest here is to find the optimal value function

$$
\phi(t, r, y)=\operatorname{Sup}_{u} \phi_{u}(t, r, y)
$$

and the optimal strategy $u^{*}=u_{s}^{*}$ such that

$$
\phi_{u^{*}}(t, r, y)=\phi(t, r, y) \text {. }
$$

\subsection{Legendre transformation}

The Legendre transform and dual theory help to transform the nonlinear partial differential equation that is formed due to (2.1.1), to a linear partial differential equation.

Theorem 2.2. (Jonsson and Sircar [11]) Let $f: R^{n} \rightarrow R$ be a convex function for $z>0$. Then the Legendre transform is defined as

$$
L(z)=\max _{y}\{f(y)=z y\}
$$

where $L(z)$ is the Legendre dual of $f(y)$. 
Since $f(y)$ is convex, from Theorem 2.2 we defined the Legendre transform

$$
\hat{\phi}(t, r, z)=\operatorname{Sup}\{\phi(t, r, y)-z y: 0<y<\infty\}, \quad 0<t<T
$$

where $\hat{\phi}$ is the dual of $\phi$ and $z>0$ is the dual variable of $x$.

The value of $y$ where this optimum is attained is denoted by $h(t, r, z)$ so that

$$
h(t, r, z)=\inf \{y: \phi(t, r, y) \geq z y+\hat{\phi}(t, r, z)\}, \quad 0<t<T .
$$

The functions $h$ and $\hat{\phi}$ are closely related and can be referred to as the dual of $\phi$. These functions are related as follows

$$
\hat{\phi}(t, r, z)=\phi(t, r, h)-z h,
$$

where

$$
h(t, r, z)=y, \quad \phi_{y}=z, \quad h=-\hat{\phi}_{z} .
$$

At terminal time, $T$, we denote

$$
\hat{U}(z)=\operatorname{Sup}\{U(y)-z y: 0<y<T\}, \quad \text { and } \quad \phi(z)=\operatorname{Sup}\{y: U(y) \geq z y+\hat{U}(z)\} .
$$

As a result

$$
\phi(z)=U^{-1}(z)
$$

where $\phi$ is the inverse of the marginal utility $U$ and note that $\phi(T, r, y)=U(y)$.

At terminal time $T$, we can define

$$
h(T, r, y)=\inf _{y>0}\{y: U(y) \geq z y+\hat{\phi}(t, r, z)\} \text { and } \hat{\phi}(t, r, z)=\operatorname{Sup}_{y>0}\{U(y)-z y\}
$$

so that $h(T, r, z)=U^{-1}(z)$.

\section{The Model}

This session introduces the financial market and proposes the optimization problems in the decumulation phase.

\subsection{The financial market}

Here, we consider a financial market that consists of a risk-free asset (i.e., cash in the bank) and a risky asset (stock). 
Let the risk-free asset, $C_{t}$, say, at any positive time, $t$, evolve thus;

$$
d C_{t}=r C_{t} d t
$$

where $r$ represents constant rate of interest.

Next, we denote the price of the risky asset (stock) at any positive time, $t$, by $S_{t}$, as in Gao [10], Akpanibah et al. [1], and Njoku et al. [12] thus;

$$
d S_{t}=\mu S_{t} d t+k S_{t}^{\beta+1} d W_{t}
$$

where $\mu(\mu>r)$ represents the instantaneous rate of return on stock, $\beta(\beta \leq 0)$ is the elastic constant parameter, $k$ is a constant, $k S_{t}^{\beta}$ represents the instantaneous volatility.

Let $\left\{W_{t} ; t \geq 0\right\}$ denote a standard Brownian motion, defined on a probability space, $(\Omega, F, P)$, where $F=\left\{F_{t}\right\}$ is an augmented filtration generated by the Brownian motion.

\subsection{Model assumption}

Consistent with the Nigerian Pension Reform Act of 2004 [13], we make the following assumptions

(a) The pension scheme accumulates wealth.

(b) There are different categories of contributions.

(c) The contributions will not willingly withdraw from the scheme.

(d) Payments are made to the retirees.

(e) An accumulated amount is paid to the next-of-kin of the dead contributors, at the instance of death by any contributor(s).

(f) A certain amount is retained from the payment made to the families of dead contributors, by the pension managers (i.e., management fee).

\subsection{Model formation (i.e., the optimization program)}

The fund accruing from the contributors can be invested in both bank and stock. Particularly, the fund to be invested by the fund manager is the surplus, which is the fund that is available after each period of routine disbursements. That is, let the contribution process be 


$$
d y=\left(1+\theta_{i}\right) C_{i+1} d t
$$

and the payment process

$$
d j=b_{i+1} d t+\left(a_{i+1}-\eta\right) d W_{s}
$$

Then the surplus

$$
\begin{aligned}
d P & =d y-d j=\left(1+\theta_{i}\right) C_{i+1} d t-\left[b_{i+1} d t+\left(a_{i+1}-\eta\right) d W_{s}\right] \\
& =\left(C_{i+1}+\theta_{i} C_{i+1}-b_{i+1}\right) d t-\left(a_{i+1}-\eta\right) d W_{s} .
\end{aligned}
$$

Therefore our task here is to construct an optimal investment strategy for the assets for the remaining periods after retirement, to enable us maximize the expected utility at each retirement period.

Without loss of generality, the pension wealth is denoted by $Y(t)$ at any time $0<t<T<T+N$, and it evolves stochastically, thus:

$$
\begin{aligned}
d Y(t)= & u_{s} Y(t) \frac{d S_{t}}{S_{t}}+\left(1-u_{s}\right) Y(t) \frac{d C_{t}}{C_{t}}+\left(C_{i+1}+\theta_{i} C_{i+1}-b_{i+1}\right) d t \\
& -\left(a_{i+1}-\eta\right) d W_{s},
\end{aligned}
$$

$i=0,1, \ldots, n-1$ and $\theta_{i}=0, \theta_{1}=1, \theta_{2}=2, \ldots, \theta_{i}$ (an integer) $=$ staff loading, where; $a_{i+1}>0$ represents various amount that is paid to the next-of-kin of the dead contributors, $b_{i+1}>0$ represents various amount paid to retired contributors, $c_{i+1}>0$ represents various amount contributed, $\eta$ is the service charge deducted from the $a_{i+1}$.

However, relevant to the provisions of the Nigerian Pension Reform Act of 2004 [13], on the eligibility condition for signing up on the pension scheme, by both government and private sectors, we have;

$$
\begin{aligned}
d Y(t)= & u_{s} Y(t) \frac{d S_{t}}{S_{t}}+\left(1-u_{s}\right) Y(t) \frac{d C_{t}}{C_{t}}+\left(C_{i+1}+\theta_{i} C_{i+1}-b_{i+1}\right) \\
& -\left(a_{i+1}-\eta\right) d W_{s}
\end{aligned}
$$

$i=4,5, \ldots, n-1$, and $\theta_{4}=4, \theta_{5}=5, \theta_{6}=6, \ldots, \theta_{n}=n$ (a positive integer) $=$ staff loading.

Assuming, $b_{i+1}=C_{i+1}+r_{d} C_{i+1} ; \quad r<r_{d}$, and $r_{d}$ represents discounted interest, 
then

$$
d Y(t)=u_{s} Y(t) \frac{d S_{t}}{S_{t}}+\left(1-u_{s}\right) Y(t) \frac{d C_{t}}{C_{t}}+\left(\theta_{i}-r_{d}\right) C_{i+1} d t-\left(a_{i+1}-\eta\right) d W_{s},
$$

$i=4,5, \ldots, n-1$, and $\theta_{4}=4, \theta_{5}=5, \theta_{6}=6, \ldots, \theta_{n}=n, \theta_{i}>0$ (integer) $=$ staff loading.

Taking into (3.1.1), (3.1.2) and (3.3.6), one obtains the wealth process

$$
\begin{aligned}
d Y(t)= & u_{s} Y(t)\left[\mu d t+k S^{\beta} d W_{t}\right]+\left(1-u_{s}\right) Y(t) r d t+\left(\theta_{i}-r_{d}\right) C_{i+1} d t-\left(a_{i+1}-\eta\right) d W_{s} \\
= & u_{s} Y(t) \mu d t+u_{s} Y(t) k S^{\beta} d W_{t}+Y(t) r d t+u_{s} Y(t) r d t+\left(\theta_{i}-r_{d}\right) C_{i+1} d t \\
& -\left(a_{i+1}-\eta\right) d W_{s} \\
= & \left(u_{s} Y(t) \mu+Y(t) r-u_{s} Y(t) r+\left(\theta_{i}-r_{d}\right) C_{i+1}\right) d t+u_{s} Y(t) k S^{\beta} d W_{t} \\
& -\left(a_{i+1}-\eta\right) d W_{s},
\end{aligned}
$$

$i=4,5, \ldots, n-1$, and $\theta_{4}=4, \theta_{5}=5, \theta_{6}=6, \ldots, \theta_{n}=n, \theta_{i}>0$ (integer) $=$ staff loading.

Based on the wealth process in (3.3.7), the pension manager seeks a strategy, $u_{t}^{*}$, which maximizes the utility function, such that $u_{t}^{*}=\max E(U(Y(T))), \forall u(t)$. Where $u(\bullet)$ is an increasing concave utility function, which satisfies the Inada conditions;

$$
U^{\prime}(+\infty)=0 \text {, and } U^{\prime}(0)=+\infty \quad(\text { cf. Gao [10]). }
$$

\section{Applying the Associated HJB Equation to Maximize Equation (3.3.7)}

Applying the associated HJB to (3.3.7), one obtains

$$
\begin{aligned}
& \varphi_{t}+\mu S \varphi_{s}+Y(t)\left[u_{s}(\mu-r)+r\right] \varphi_{y}+\left(\theta_{i}-r_{d}\right) C_{i+1} \varphi_{y}+\frac{1}{2} k^{2} S^{2 \beta+2} \varphi_{s s} \\
& +\frac{1}{2} U_{s}^{2} Y^{2} k^{2} S^{2 \beta} \varphi_{y y}-\frac{1}{2} \alpha \gamma U s Y(t) k S^{\beta} a_{i+1} \varphi_{y y}+\frac{1}{2} \alpha \gamma u_{s} Y(t) k S^{\beta} \eta \varphi_{y y} \\
& +\frac{1}{2}\left(a_{i+1}-\eta\right)^{2} \varphi_{y y}+\frac{1}{2} \alpha \gamma k^{2} S^{\beta+2} Y(t) u_{s} \varphi_{s y}-\frac{1}{2} \alpha \gamma k S^{\beta+1} a_{i+1} \varphi_{s y}
\end{aligned}
$$


$+\frac{1}{2} \alpha \gamma k S^{\beta+1} \eta \varphi_{s y}=0$.

To obtain the optimal value $u_{s}^{*}$, we differentiate (4.1) with respect to $u_{s}$

$$
\begin{aligned}
& \varphi_{t}+\mu s \varphi_{s}+\left(\theta_{i}-r_{d}\right) C_{i+1} \varphi_{y}+\frac{1}{2} k^{2} s^{2 \beta+2} \varphi_{s s}+\frac{1}{2}\left(a_{i+1}-\eta\right)^{2} \varphi_{y y} \\
& -\frac{1}{2} \alpha \gamma k s^{\beta+1} a_{i+1} \varphi_{s y}+\frac{1}{2} \alpha \gamma k s^{\beta+1} \eta \varphi_{s y} \\
& +\sup _{u_{s}}\left\{\begin{array}{l}
Y(t)\left(u_{s}(\mu-r)+r\right) \varphi_{y}+\frac{1}{2} u_{s}^{2} Y(t)^{2} k^{2} S^{2 \beta} \varphi_{y y} \\
-\frac{1}{2} \alpha \gamma u_{s} Y(t) k S^{\beta} a_{i+1} \varphi_{y y}+\frac{1}{2} \alpha \gamma u_{s} k S^{\beta} \eta \varphi_{y y} \\
+\frac{1}{2} \alpha \gamma k^{2} s^{\beta+2} Y(t) u_{s} \varphi_{s y}
\end{array}\right\}=0,
\end{aligned}
$$

so that

$$
\begin{aligned}
& \therefore u_{S}^{*}=\frac{\alpha \gamma a_{i+1}}{2 Y(t) k S^{\beta}}-\frac{\alpha \gamma \eta}{2 Y(t) k S^{\beta}}-\frac{(\mu-r)}{Y(t) k^{2} S^{2 \beta}} \frac{\varphi_{y}}{\varphi_{y y}}-\frac{\alpha \gamma}{2 Y(t) S^{\beta-2}} \cdot \frac{\varphi_{s y}}{\varphi_{y y}} \\
& \Rightarrow u_{S}^{*}=\frac{\alpha \gamma\left(a_{i+1}-\eta\right)}{2 Y(t) k S^{\beta}}-\frac{(\mu-r)}{Y(t) k^{2} S^{2 \beta}} \frac{\varphi_{y}}{\varphi_{y y}}-\frac{\alpha \gamma}{2 Y(t) S^{\beta-2}} \cdot \frac{\varphi_{s y}}{\varphi_{y y}} .
\end{aligned}
$$

Putting back (4.3) into (4.2) gives;

$$
\begin{aligned}
& \varphi_{t}+\mu s \varphi_{s}+\left[Y(t) r+\left(\theta_{i}-r_{d}\right) C_{i+1}+\frac{\alpha \gamma(\mu-r)\left(a_{i+1}-\eta\right)}{2 k S^{\beta}}\right] \varphi_{y} \\
& +\frac{1}{2} k^{2} S^{2 \beta+2} \varphi_{s s}+c_{1} \varphi_{y y}+\left[\frac{(\alpha \gamma)^{2} k S^{2}\left(a_{i+1}-\eta\right)}{4}-\frac{\alpha \gamma k S^{\beta+1}\left(a_{i+1}-\eta\right)}{2}\right] \varphi_{s y} \\
& -\frac{(\mu-r)^{2} \varphi_{y}^{2}}{2 k^{2} S^{2 \beta} \varphi_{y y}}-\frac{\alpha \gamma(\mu-r) \varphi_{y} \varphi_{s y}}{2 S^{\beta-2} \varphi_{y y}}-\frac{k^{2} S^{4} \varphi_{s y}^{2}(\alpha \gamma)^{2}}{8 \varphi_{y y}}=0,
\end{aligned}
$$

$i=4,5, \ldots, n-1, r<r_{d}$ and $\theta_{4}=4, \theta_{5}=5, \theta_{6}=6, \ldots, \theta_{n}=n, \theta_{n-1}=n-1, \theta_{i}>0$ (integer) $=$ staff loading, and

$$
c_{1}=\frac{1}{2}\left(a_{i+1}-\eta\right)^{2}+\frac{3}{4}(\alpha \gamma)^{2}\left(a_{i+1}-\eta\right)^{2} .
$$


Having seen that stochastic control problem described in the previous session has been converted to a nonlinear PDE, our next tax is to solve for $\varphi$ in (4.4) and subsequently substitute it into (4.3), to enable us obtain the optimal wealth investment strategy (i.e., the control strategy). In order to achieve this, we use employ the services of the Dual theory and Legendre transformation.

\section{Transforming (4.4) into its Dual and Applying Legendre Transformation}

Here, we transform the nonlinear second order partial differential equation (4.4) into a linear PDE, using the Dual theory and Legendre transformations in Gao [10], that is;

$$
\phi_{y}=z \text { and } \phi_{t}=\hat{\phi}_{t}, \phi_{s}=\hat{\phi}_{s}, \phi_{s s}=\hat{\phi}_{s s}-\frac{\hat{\phi}_{s z}^{2}}{\hat{\phi}_{z z}}, \phi_{y y}=\frac{-1}{\hat{\phi}_{z z}}, \phi_{y s}=\frac{-\hat{\phi}_{s z}}{\hat{\phi}_{z z}} \text {. }
$$

Putting (5.1) into (4.4)

$$
\begin{aligned}
& \hat{\varphi}_{t}+\mu S \hat{\varphi}_{s}+\left[Y(t) r+\left(\theta_{i}-r_{d}\right) C_{i+1}+\frac{\alpha \gamma(\mu-r)}{2 k S^{\beta}}\left(a_{i+1}-\eta\right)\right] z \\
& +\frac{1}{2} k^{2} S^{2 \beta+2} \hat{\varphi}_{s s}-\frac{c_{1}}{\hat{\varphi}_{z z}} \\
& -\left[\frac{(\alpha \gamma)^{2} k S^{2}\left(a_{i+1}-\eta\right)}{4}-\frac{\alpha \gamma k S^{\beta+1}\left(a_{i+1}-\eta\right)}{2}\right] \frac{\hat{\varphi}_{s z}}{\hat{\varphi}_{z z}} \\
& +\frac{(\mu-r)^{2}}{2 k^{2} S^{2 \beta}} z^{2} \hat{\varphi}_{z z}-\frac{\alpha \gamma(\mu-r)}{2 s^{\beta-2}} z \hat{\varphi}_{s z}+\frac{(\alpha \gamma)^{2} k^{2} S^{4}}{8} \frac{\hat{\varphi}_{s z}^{2}}{\hat{\varphi}_{z z}}=0
\end{aligned}
$$

$i=4,5, \ldots, n-1, r<r_{d}$ and $\theta_{4}=4, \theta_{5}=5, \theta_{6}=6, \ldots, \theta_{n}=n, \theta_{n-1}=n-1, \theta_{i}>0$ (integer) $=$ staff loading, and $c_{1}=\frac{1}{2}\left(a_{i+1}-\eta\right)^{2}+\frac{3}{4}(\alpha \gamma)^{2}\left(a_{i+1}-\eta\right)^{2}$.

Taking $y=h=-\hat{\phi}_{z}$ into (5.2), and differentiating with respect to $z$, we obtain

$$
\begin{aligned}
& h_{t}+\mu S h_{s}-r z h_{z}-r h-\left(\theta_{i}-r_{d}\right) C_{i+1}-\frac{\alpha \gamma(\mu-r)\left(a_{i+1}-\eta\right)}{2 k S^{\beta}} \\
& +\frac{1}{2} k^{2} S^{2 \beta+2} h_{s s}+\frac{c_{1} h_{z z}}{h_{z}^{2}}
\end{aligned}
$$




$$
\begin{aligned}
& +\left[\frac{(\alpha \gamma)^{2} k S^{2}\left(a_{i+1}-\eta\right)}{4}-\frac{\alpha \gamma k S^{\beta+1}\left(a_{i+1}-\eta\right)}{2}\right] \frac{h_{s}}{h_{z}}-\frac{\alpha \gamma(\mu-r)}{2 S^{\beta-2}} h_{s} \\
& -\frac{\alpha \gamma(\mu-r)}{2 S^{\beta-2}} z h_{s z}+\frac{(\mu-r)^{2}}{k^{2} S^{2 \beta}} z h_{z}+\frac{(\mu-r)^{2}}{2 k^{2} S^{2 \beta}} z^{2} h_{z z} \\
& +\frac{(\alpha \gamma)^{2} k^{2} S^{4}}{8} \frac{h_{s z} h_{s}}{h_{z}}-\frac{(\alpha \gamma)^{2} k^{2} S^{4} h_{s}^{2} h_{z z}}{8 h_{z}^{2}}=0,
\end{aligned}
$$

where

$$
c_{1}=\frac{1}{2}\left(a_{i+1}-\eta\right)^{2}+\frac{3}{8}(\alpha \gamma)^{2}\left(a_{i+1}-\eta\right)^{2},
$$

with,

$$
\begin{aligned}
u_{s}^{*} & =\frac{\alpha \gamma\left(a_{i+1}-\eta\right)}{2 Y(t) k S^{\beta}}+(\mu-r) \frac{z}{\hat{\varphi}_{z z}}-\frac{\alpha \gamma}{2 Y(t) S^{\beta-2}} \hat{\varphi}_{s z} \\
\Rightarrow u_{s}^{*} & =\frac{\alpha \gamma\left(a_{i+1}-\eta\right)}{2 Y(t) k S^{\beta}}-(\mu-r) \frac{z}{h_{z}}-\frac{\alpha \gamma}{2 Y(t) S^{\beta-2}} h_{s} .
\end{aligned}
$$

\section{Test for Some Utility Functions}

Here, we seek to obtain the explicit solutions for the CRRA and CARA utility functions, using change of variable method.

\subsection{Explicit solution to the CRRA utility}

Following Gao [10] and Zhang and Rong [5], we use

$$
h(t, s, z)=z^{\frac{1}{p-1}}, \quad p<1, p \neq 0 .
$$

Let us conjecture a solution to (5.3), thus

$$
h(t, s, z)=z^{\frac{1}{p-1}} g(t, s)+a(t) ; a(T)=0 ; g(T, s)=1 .
$$

Now, obtaining the various first and second partial derivatives with respect to $t, s, z$, we have 


$$
\begin{aligned}
& h_{t}=g_{t} z^{\frac{1}{p-1}}+a^{\prime}(t) ; h_{s}=g_{s} z^{\frac{1}{p-1}} ; h_{z}=\frac{-g}{1-p} z^{\frac{1}{p-1}-1} ; h_{s s}=g_{s s} z^{\frac{1}{p-1}} \\
& h_{s z}=\frac{-g}{1-p} z^{\frac{1}{p-1}-1} ; h_{z z}=\frac{(2-p) g}{(1-p)^{2}} z^{\frac{1}{p-1}-2}=0 .
\end{aligned}
$$

Putting (6.1.2) and (6.1.3) into (5.3), we obtain

$$
\begin{aligned}
& g_{t} z^{\frac{1}{p-1}}+a^{\prime}(t)+\mu s g_{s} z^{\frac{1}{p-1}}+\frac{r g z^{\frac{1}{p-1}}}{1-p}-r g z^{\frac{1}{p-1}}-r a(t)-\left(\theta_{i}-r_{d}\right) C_{i+1} \\
& -\frac{\alpha \gamma(\mu-r)\left(a_{i+1}-\eta\right)}{2 k s^{\beta}}+\frac{1}{2} k^{2} s^{2 \beta+2} g_{s s} z^{\frac{1}{p-1}}+\frac{c_{1}(2-p)}{g} \frac{1}{z^{\frac{1}{p-1}}} \\
& -\left[\frac{(\alpha \gamma)^{2} k s^{2}\left(a_{i+1}-\eta\right)}{4}-\frac{\alpha \gamma k s^{\beta+1}\left(a_{i+1}-\eta\right)}{2}\right] \frac{g_{s}(1-p) z}{g} \\
& -\frac{\alpha \gamma(\mu-r)}{2 s^{\beta-2}} g_{s} z^{\frac{1}{p-1}}+\frac{\alpha \gamma(\mu-r) g_{s} z^{\frac{1}{p-1}}}{2 s^{\beta-2}(1-p)}-\frac{(\mu-r)^{2} g}{k^{2} s^{2 \beta}(1-p)} z^{\frac{1}{p-1}} \\
& +\frac{(\mu-r)^{2}(2-p) g}{2 k^{2} s^{2 \beta}(1-p)^{2}} z^{\frac{1}{p-1}}+\frac{(\alpha \gamma)^{2} k^{2} s^{4} g_{s}^{2} z^{\frac{1}{p-1}}}{8} \\
& -\frac{k^{2} s^{4} g_{s}^{2} z^{\frac{1}{p-1}}(2-p)(\alpha \gamma)^{2}}{8 g}=0
\end{aligned}
$$

Factoring out terms that depend on $z^{\frac{1}{p-1}}$, and $z$, and the ones that is independent of either of the two mentioned, we split (6.1.4) into three, thus

$$
a^{\prime}(t)-r a(t)-\left(\theta_{i}-r_{d}\right) C_{i+1}-\frac{\alpha \gamma(\mu-r)\left(a_{i+1}-\eta\right)}{2 k s^{\beta}}=0, \quad r<r_{d},
$$




$$
\left[\frac{(\alpha \gamma)^{2} k s^{2}\left(a_{i+1}-\eta\right)}{4}-\frac{\alpha r k s^{\beta+1}(\mu-r)\left(a_{i+1}-\eta\right)}{2}\right] \frac{g_{s}(1-p)}{g}=0, \quad g \neq 0
$$

and

$$
\begin{aligned}
& g_{t}+\mu s g_{s}+\frac{r g}{1-p}-r g+\frac{1}{2} k^{2} s^{2 \beta+2} g_{s s}+\frac{g}{c_{1}(2-p)} \\
& -\frac{\alpha r(\mu-r) g_{s}}{2 s^{\beta-2}}+\frac{\alpha \gamma(\mu-r) g_{s}}{2 s^{2 \beta-2}(1-p)}-\frac{(\mu-r)^{2} g}{k^{2} s^{2 \beta}(1-p)} \\
& +\frac{(\mu-r)^{2}(2-p) g}{2 k^{2} s^{2 \beta}(1-p)^{2}}-\frac{(\alpha \gamma)^{2} k^{2} s^{4} g_{s}^{2}}{8}+\frac{k^{2} s^{4} g_{s}^{2}}{8 g}(2-p)(\alpha \gamma)^{2}=0, \quad g \neq 0 .
\end{aligned}
$$

Solving (6.1.5) at the boundary condition, $a(T)=0$, we obtain the continuous annuity of duration, $T-t$, yields

$$
a(t)=-[T-t] \ell^{-r t}\left[\left(\theta_{i}-r_{d}\right) C_{i+1} 2 k+\alpha \gamma u_{s}\left(a_{i+1}-\eta\right)\right],
$$

where $a(t)=-[T-t]\left[\left(\theta_{i}-r_{d}\right) C_{i+1} 2 k+\alpha \gamma u_{s}\left(a_{i+1}-\eta\right)\right]$, and $\ell^{-r t}$ are the so called continuous annuity of duration $T-t$, and the continuous technical rate, respectively.

From (6.1.6), we propose the following

Proposition 6.1.1. Let, (a) $d w_{s} d w_{t}=\frac{1}{2} \alpha \gamma d t ; \frac{\alpha \gamma}{2} \neq 1$ (b) $\beta \neq 1$. Then

$$
\left[\frac{(\alpha \gamma)^{2} k s^{2}\left(a_{i+1}-\eta\right)^{2}}{4}-\frac{\alpha \gamma k s^{\beta+1}\left(a_{i+1}-\eta\right)}{2}\right] \frac{g_{s}(1-p)}{g}=0 ; \quad p<1 .
$$

Proof. Suppose, for contradiction, $\beta=1, \frac{\alpha \gamma}{2}=1$. We observe that either of the following two cases arises

Case 1: $\frac{g_{s}(1-p)}{g}=0 \Rightarrow p=1 \Rightarrow \Leftarrow p<1$, by definition of $p$ in (6.1.1).

Case 2: $\frac{(\alpha \gamma)^{2} k s^{2}\left(a_{i+1}-\eta\right)^{2}}{4}=\frac{\alpha \gamma k s^{\beta+1}\left(a_{i+1}-\eta\right)}{2} \Rightarrow \frac{\alpha \gamma s^{2}}{2}=s^{\beta+1}$. 
But $s^{2}=s^{\beta+1}$, whenever $\frac{\alpha \gamma}{2}=1$, satisfied by the supposition above $\Rightarrow \beta=1$ whenever $\frac{\alpha \gamma}{2}=1$, by law of indices. But by Proposition 6.1.1, $d w_{s} d w_{t}=\frac{1}{2} \alpha \gamma d t$ $\Rightarrow \frac{\alpha \gamma}{2}=d w_{s} d w_{t} \neq 1 \Rightarrow \Leftarrow$, contradicting the assumption that $\frac{\alpha \gamma}{2}=1$.

Hence, the proposition holds, and this completes the proof.

Corollary 6.1.1. The correlation between stock and time, represented, $d w_{s} d w_{t}$ is equal to 1 , thereby making $\frac{\alpha \gamma}{2}=1$, holds only if stock attains its maximum yield at every increase in time, which is not realistic.

Lastly, solving (6.1.7), observe firstly that the equation contains some variable coefficients, $s, s^{2 \beta+2}, s^{-2 \beta}, s^{-(\beta-2)}$, and this makes obtaining solution somewhat difficult. However, in order to overcome this difficulty, we employ the services of power transformation and change of variable technique as in Cox et al. [6].

Assuming

$$
g(t, s)=f(t, j), \quad j=s^{-2 \beta}
$$

such that

$$
g_{t}=j_{t} ; g_{s}=-2 \beta s^{-(2 \beta+1)} f_{j} ; g_{s s}=4 \beta^{2} s^{-2(2 \beta+1)} f_{j j}+2 \beta(2 \beta+1) s^{-2(\beta+1)} f_{j},
$$

then putting (6.1.10) and (6.1.11) into (6.1.7), gives

$$
\begin{aligned}
& f_{t}-\mu 2 \beta j f_{t}+\frac{r}{1-p} f-r f+k^{2} 2 \beta^{2} j f_{j j}+k^{2} \beta(2 \beta+1) f_{j}+\frac{1}{c_{1}(2-p)} \cdot f \\
& +\frac{(\mu-r) 4 \beta j}{\alpha \gamma s^{2}} \cdot f_{t}-\frac{(\mu-r) 4 \beta j}{\alpha \gamma s^{2}(1-p)} \cdot f_{t} \\
& -\frac{(\mu-r)^{2}}{k^{2}(1-p)} \cdot f+\frac{(\mu-r)^{2}(2-p) j}{2 k^{2}(1-p)^{2}} \cdot f-k^{2} \beta^{2} j^{2} f_{t}^{2}+\frac{2 k^{2} \beta^{2} j^{2}}{f} \cdot f_{t}^{2}=0,
\end{aligned}
$$

where $s^{\beta}=\frac{\alpha \gamma}{2} \cdot s$. 
Following Proposition 6.1.1, we observe that some cases may arise in (6.1.12), $\beta=0, \beta<1$, provided $p \neq 0, p<1$.

Case 1: $\beta=0$ (i.e., the GBM case), yields

$$
\begin{array}{r}
f_{t}+\left[\frac{r}{1-p}-r+\frac{1}{c_{1}(2-p)}-\frac{(\mu-r)^{2}}{k^{2}(1-p)}+\frac{2(\mu-r)^{2}(2-p)}{k^{2}(1-p)^{2}(\alpha \gamma)^{2} s^{2}}\right] \cdot f=0 \\
\beta<-1, p \neq 0 .
\end{array}
$$

Next, we find the solution of (6.1.13), using the structure below

$$
f(t, j)=A(t) \ell^{B(t) j} ; A(T)=1, B(T)=0 \Rightarrow f_{t}=A_{t} \ell^{B(t) j}+A(t) B_{t} j \ell^{B(t) j} .
$$

Putting (6.1.14) into (6.1.13) yields

$$
\frac{A_{t}}{A(t)}+B_{t} j+\frac{r}{1-p}-r+\frac{1}{c_{1}(2-p)}-\frac{(\mu-r)^{2}}{k^{2}(1-p)}+\frac{(\mu-r)^{2}(2-p) j}{2 k^{2}(1-p)^{2}}=0
$$

where $s^{\beta}=\frac{\alpha \gamma}{2} \cdot s$ is re-substituted and $j=s^{-2 \beta}$.

Observe that we can further split (6.1.15) by separating the terms that depends on $j$, thus

$$
B_{t}+\frac{(\mu-r)^{2}(2-p)}{2 k^{2}(1-p)^{2}}=0
$$

and

$$
\frac{A_{t}}{A(t)}+\frac{r}{1-p}-r+\frac{1}{c_{1}(2-p)}-\frac{(\mu-r)^{2}}{k^{2}(1-p)}=0 .
$$

Solving (6.1.16) at the initial condition $B(T)=0$, yields

$$
B(t)=[T-t]\left(\frac{u_{S}^{2}(2-p)}{2 k^{2}(1-p)}\right)
$$

where $\mu=u_{s}+r$. 
Next, we solve (6.1.17) at initial condition $A(T)=1$, yields

$$
A(t)=\ell^{\left[\frac{r}{1-p}-r+\frac{1}{c_{1}(2-p)}-\frac{u_{s}^{2}}{k^{2}(1-p)}\right][T-t]}, \quad p \neq 0, p<-1,
$$

where $\mu=u_{s}+r$.

Taking into (6.1.18), (6.1.19) and (6.1.14), we have

$$
f(t, j)=\ell^{\left[\frac{r}{1-p}-r+\frac{1}{c_{1}(2-p)}-\frac{u_{s}^{2}}{k^{2}(1-p)}+\frac{u_{s}^{2}(2-p) j}{2 k^{2}(1-p)}\right][T-t]} .
$$

In this sequel, we state;

Theorem 6.1. Let equations (6.1.2), (6.1.8), (6.1.10) and (6.1.20) hold. Then the optimal wealth investment made in stock is given as

$$
u_{s}^{*}=\frac{\alpha \gamma\left(a_{i+1}-\eta\right)}{2 Y(t) k}-u_{s}(p-1) z^{\frac{2 p-3}{p-1}} \ell-\left[\frac{r p}{1-p}-r+\frac{1}{c_{1}(2-p)}-\frac{u_{s}^{2}}{k^{2}(1-p)}+\frac{u_{s}^{2}(2-p) j}{2 k^{2}(1-p)}\right][T-t] \text {. }
$$

Proof. Taking into (6.1.8), (6.1.10), (6.1.20) and (6.1.2), we have

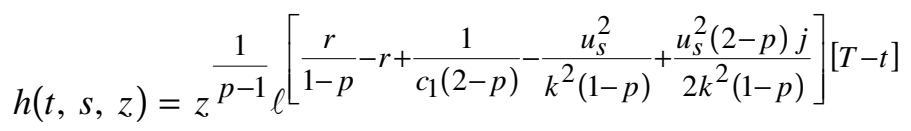

$$
\begin{aligned}
& -\ell^{-r t}\left[2 k\left(\theta_{i}-r_{d}\right) C_{i+1}+\alpha \gamma u_{s}\left(a_{i+1}-\eta\right)\right][T-t] .
\end{aligned}
$$

Differentiating (6.1.21) w.r.t. $s$ and $z$, yields

$$
h_{s}=0
$$

and

$$
h_{z}=\frac{1}{p-1} z^{\frac{1}{p-1}-1} \ell^{\left[\frac{r}{1-p}-r+\frac{1}{c_{1}(2-p)}-\frac{u_{s}^{2}}{k^{2}(1-p)}+\frac{u_{s}^{2}(2-p) j}{2 k^{2}(1-p)}\right][T-t]} .
$$

Therefore, taking into (6.22), (6.23) and (5.5), thus

$$
u_{s}^{*}=\frac{\alpha \gamma\left(a_{i+1}-\eta\right)}{2 Y(t) k}
$$




$$
-u_{s}(p-1) z^{\frac{2 p-3}{p-1}} \ell-\left[\frac{r p}{1-p}-r+\frac{1}{c_{1}(2-p)}-\frac{u_{s}^{2}}{k^{2}(1-p)}+\frac{u_{s}^{2}(2-p) j}{2 k^{2}(1-p)}\right][T-t]
$$

where $c_{1}=\frac{1}{2}\left(a_{i+1}-\eta\right)^{2}+\frac{3}{8}(\alpha \gamma)^{2}\left(a_{i+1}-\eta\right)^{2}$, as required.

\subsection{Explicit solution for the CARA utility}

Suppose, the plan number takes an exponential utility function

$$
U(y)=-\frac{1}{q} e^{-q y}, \quad q>0 .
$$

The decision maker's absolute risk aversion, presented in (6.2.1) is constant, and is called CARA utility.

Combining $h(T, s, z)=\left(U^{\prime}\right)^{-1}(z)$ and (6.2.1) above, we have

$$
h(T, s, z)=-\frac{1}{q} h z
$$

Let us now conjecture a solution to (5.3), thus

$$
h(T, s, z)=-\frac{1}{q}[h(t)(h z+m(t, s))]+a(t)
$$

such that $h(T)=1 ; a(T)=0 ; m(T, s)=0$.

Obtaining the first, second and mixt derivative of (6.2.3) with respect to $t, s, z$

$$
\begin{aligned}
& h_{t}=-\frac{1}{q}\left[b^{\prime}(t)(\ln z+m(t, s))+b(t) m_{t}\right]+a^{\prime}(t) ; h_{s}=-\frac{1}{q} h(t) m_{s} ; h_{z}=\frac{-b(t)}{q^{2}} \\
& h_{z z}=\frac{b(t)}{q z^{2}} ; h_{s s}=-\frac{1}{q} b(t) m_{s s} ; h_{s z}=0 .
\end{aligned}
$$

Putting (6.2.4) into (5.3) yields

$$
\begin{aligned}
& b^{\prime}(t) \ln z+b^{\prime}(t) m(t, s)+b(t) m_{t}+a^{\prime}(t) q-\mu s b(t) m_{s}+\frac{r z b(t)}{q}+r b(t) \ln z \\
& +r b(t) m(t, s)+r a(t) q-\left(\theta_{i}-r\right) C_{i+1} q-\frac{\alpha \gamma(\mu-r)\left(a_{i+1}-\eta\right)}{s^{\beta}} q
\end{aligned}
$$




$$
\begin{aligned}
& -\frac{k^{2} s^{2 \beta+2} b(t)}{2} m_{s s}+\frac{c_{1} q^{4}}{z^{2} b(t)}+\frac{(\alpha \gamma)^{2} k s^{2}\left(a_{i+1}-\eta\right)}{4} m_{s} q^{2} \\
& -\frac{\alpha \gamma k s^{\beta+1}\left(a_{i+1}-\eta\right)}{2} m_{s} q^{2}+\frac{\alpha \gamma(\mu-\gamma) b(t) m_{s}}{2 s^{\beta-2}}-\frac{(\mu-\gamma)^{2} z b(t)}{k^{2} s^{2 \beta} q}+\frac{(\mu-\gamma)^{2} b(t)}{2 k^{2} s^{2 \beta}} \\
& +\frac{k^{2} s^{4} m_{s}^{2} b(t) q^{2}}{8 z^{2}}(\alpha \gamma)^{2}=0 .
\end{aligned}
$$

Splitting (6.2.5) into three in order to remove dependency on $\ln z, q$ and $h(t)$ gives

$$
\begin{gathered}
b^{\prime}(t)+r b(t)=0, \\
a^{\prime}(t)+r a(t)-\left(\theta_{i}-r_{d}\right) C_{i+1}-\frac{\alpha \gamma(\mu-r)\left(a_{i+1}-\eta\right)}{2 k s^{\beta}}=0
\end{gathered}
$$

and

$$
\begin{aligned}
& \frac{b^{\prime}(t) m(t, s)}{b(t)}+m_{t}-\mu s m_{s}+\frac{r z}{q}+r m(t, s)-\frac{k^{2} s^{2 \beta+2}}{2} m_{s s} \\
& +\frac{c_{1} q^{4}}{z^{2} b^{2}}+\frac{(\alpha \gamma)^{2} k s^{2}\left(a_{i+1}-\eta\right)}{4} m_{s} q-\frac{\alpha \gamma k s^{\beta+1}\left(a_{i+1}-\eta\right)}{2} m_{s} q+\frac{\alpha \gamma(\mu-r) m_{s}}{2 s^{\beta-2}} \\
& -\frac{(\mu-r)^{2} z}{k^{2} s^{2 \beta} q}+\frac{(\mu-r)^{2}}{2 k^{2} s^{2 \beta}}+\frac{k^{2} s^{4} m_{s}^{2} q^{2}}{8 z^{2}}(\alpha \gamma)^{2}=0 .
\end{aligned}
$$

Solving (6.2.6) at the initial condition, $b(T)=1$, we have

$$
b(t)=\ell^{r(T-t)} .
$$

Next, solving (6.2.7) at the initial condition, $a(T)=0$, we have

$$
\begin{aligned}
a(t)=e^{-\gamma t}\left[\int e^{r t} \frac{\left[2 k\left(\theta_{i}-r_{d}\right) C_{i+1}+\alpha \gamma u_{L}\left(a_{i+1}-\eta\right)\right]}{2 k} d t\right. \\
\left.\quad-\int e^{r T} \frac{\left[2 k\left(\theta_{i}-r_{d}\right) C_{i+1}+\alpha \gamma u_{L}\left(a_{i+1}-\eta\right)\right]}{2 k} d T\right] .
\end{aligned}
$$

More so, to solve (6.2.8), observe that (6.2.8) contains the coefficients, 
$s, s^{-(\beta-2)}, s^{-2 \beta}, s^{\beta+1}, s^{2}$. Therefore, we assume that

$$
m(t, s)=f(t, v) \ni v=s^{-2 \beta} .
$$

Obtaining the first and second derivative of (6.2.11) with respect to $t, s$, yields

$$
\begin{aligned}
& m_{t}=f_{t} ; m_{s}=-2 \beta s^{-2 \beta-1} f_{v} ; m_{s}^{2}=4 \beta^{2} s^{-4 \beta-2} f_{v}^{2} \\
& m_{s s}=2 \beta(2 \beta+1) s^{-2 \beta-2} f_{v}+4 \beta^{2} s^{-4 \beta-2} f_{v v} .
\end{aligned}
$$

Putting (6.2.11), and (6.2.12) into (6.2.8), and assuming that $\mu=u_{L}+r$, since the stock appreciates instantaneously

$$
\begin{aligned}
& \frac{b^{\prime}(t)}{b(t)} f+f_{t}+2\left(u_{L}+r\right) \beta_{v} f_{v}+\frac{r z}{q}+r f-k^{2} \beta(2 \beta+1) f_{v}-2 k^{2} \beta^{2} v f_{v v}+\frac{c_{1} q^{4}}{z^{2} b(t)^{2}} \\
& -\frac{(\alpha \gamma)^{2} k s\left(a_{i+1}-\eta\right)}{2} q v f_{v}+\alpha \gamma k s^{-\beta}\left(a_{i+1}-\eta\right) q f_{v}-\frac{\alpha \gamma u_{L} \beta v f_{v}}{s^{\beta-1}}-\frac{u_{L}^{2} z v}{k q}+\frac{u_{L}^{2}}{2 k^{2}} v \\
& +\frac{k^{2} s^{4} \beta^{2} q^{2} v^{2} f_{v}^{2}}{2 z^{2}}=0 .
\end{aligned}
$$

We also conjecture a solution of (6.2.13), thus

$$
\begin{gathered}
f(t, v)=\tilde{A}(t)+\tilde{B}(t) v ; \tilde{A}(T)=0, \tilde{B}(t)=0, \\
\Rightarrow f_{t}=\tilde{A}(t)+\tilde{B}(t) v ; f_{v}=\tilde{B}(t) ; f_{v v}=0 ; f_{v}^{2}=\tilde{B}^{2}(t) .
\end{gathered}
$$

Putting (6.2.15) and (6.2.14) into (6.2.13)

$$
\begin{aligned}
& \frac{b^{\prime}(t)}{b(t)}(\tilde{A}(t)+\tilde{B}(t) v)+\tilde{A}_{t}+\tilde{B}_{t} v+2\left(u_{L}+r\right) \beta v \tilde{B}(t)+\frac{r z}{q} \\
& +r(\tilde{A}(t)+\tilde{B}(t) v)-k^{2} \beta(2 \beta+1) \tilde{B}(t)+\frac{c_{1} q^{4}}{z^{2} b(t)^{2}}-\frac{(\alpha \gamma)^{2} k s\left(a_{i+1}-\eta\right)}{2} q \tilde{B}(t) \\
& +\alpha \gamma k s^{-\beta}\left(a_{i+1}-\eta\right) q \tilde{B}(t)-\frac{\alpha \gamma u_{L} \beta v}{s^{\beta-1}} \tilde{B}(t)-\frac{u_{L}^{2} z v}{k q}+\frac{u_{L}^{2}}{2 k^{2}} v \\
& +\frac{k^{2} s^{4} \beta^{2} q^{2} v^{2}}{2 z^{2}} \tilde{B}^{2}(t)=0 .
\end{aligned}
$$


But from (6.2.6)

$$
\frac{b^{\prime}(t)}{b(t)}=-r
$$

Putting (6.2.17) into (6.2.16), gives

$$
\begin{aligned}
& \tilde{A}_{t}+\tilde{B}_{t} v+2\left(u_{L}+r\right) \beta y \tilde{B}(t)+\frac{r z}{q}-k^{2} \beta(2 \beta+1) \tilde{B}(t)+\frac{c_{i} q^{4}}{z^{2} b^{2}(t)} \\
& -\frac{(\alpha \gamma)^{2} k s\left(a_{i+1}-\eta\right)}{2} q v \tilde{B}(t)+\alpha \gamma k s^{-\beta}\left(a_{i+1}-\eta\right) q \tilde{B}(t)-\frac{\alpha \gamma u_{L} \beta v}{s^{\beta-1}} \tilde{B}(t) \\
& -\frac{u_{L}^{2}}{2 k^{2}} v+\frac{k^{2} s^{4} \beta^{2} q^{2}}{2 z^{2}} v^{2} \tilde{B}^{2}(t)=0 .
\end{aligned}
$$

Some cases may arise $(\beta=0$ or $\beta<0)$

Case 1: $\beta=0$ (i.e., the Geometric Brownian Motion (GBM)), (6.2.18) becomes

$$
\begin{aligned}
& \tilde{A}_{t}+\tilde{B}_{t} v+\frac{r z}{q}+\frac{c_{i} q^{4}}{z^{2} b^{2}(t)}-\frac{(\alpha \gamma)^{2} k s\left(a_{i+1}-\eta\right)}{2} q v \tilde{B}(t)+\alpha \gamma k\left(a_{i+1}-\eta\right) q \tilde{B}(t) \\
& -\frac{u_{L}^{2}}{2 k^{2}} v=0 .
\end{aligned}
$$

Factoring out terms that depend on $v$ and splitting (6.2.19)

$$
\begin{aligned}
\tilde{A}_{t}+\frac{r z}{q}+\frac{c_{i} q^{4}}{z^{2} b^{2}(t)}+\alpha \gamma k\left(a_{i+1}-\eta\right) q \tilde{B}(t) & =0, \\
\tilde{A}(T) & =0, \tilde{B}(T)=0, b(T)=1, q>0
\end{aligned}
$$

and

$$
\begin{aligned}
& \left(\tilde{B}_{t}-\frac{(\alpha \gamma)^{2} k s\left(a_{i+1}-\eta\right)}{2} q \tilde{B}(t)-\frac{u_{L}^{2}}{2 k^{2}}\right) v=0 \\
\Rightarrow & \tilde{B}_{t}-\frac{(\alpha \gamma)^{2} k s\left(a_{i+1}-\eta\right)}{2} q \tilde{B}(t)-\frac{u_{L}^{2}}{2 k^{2}}=0, q>0, \tilde{B}(T)=0, s=s(t) .
\end{aligned}
$$


Equivalently

$$
\frac{d \tilde{B}}{d t}-P(t) \tilde{B}=Q(t), q>0, \tilde{B}(T)=0, s=s(t)
$$

where

$$
P(t)=\frac{(\alpha \gamma)^{2} k s(t)\left(a_{i+1}-\eta\right)}{2} q ; \quad Q(t)=\frac{u_{L}^{2}}{2 k^{2}} .
$$

Solving (6.2.22), at $\tilde{B}(T)=0$, using integrating factor method

$$
\begin{aligned}
\tilde{B}(t)= & e^{-\frac{(\alpha \gamma)^{2} k\left(a_{i+1}-\eta\right)}{2} q \int s(T) d t} \frac{u_{L}^{2}}{2 k^{2}}\left[\int e^{\frac{(\alpha \gamma)^{2} k\left(a_{i+1}-\eta\right)}{2} q \int s(t) d t} d t\right. \\
& \left.-\int e^{\frac{(\alpha \gamma)^{2} k\left(a_{i+1}-\eta\right)}{2} q \int s(T) d T} d T\right]
\end{aligned}
$$

Putting back (6.2.24) into (6.2.20) and solving at $\tilde{A}(T)=0$, gives

$$
\tilde{A}=\int w(T) d T+\int w(t) d t .
$$

Theorem 6.2. Let (6.2.9), (6.2.10) and (6.2.3) hold. Then the optimal wealth invested in stock for the CARA utility function is given by

$$
\begin{aligned}
u_{s}^{*}= & \frac{\alpha \gamma\left(a_{i+1}-\eta\right)}{2 Y(t) k S^{\beta}}+u_{L} \ell^{r(T-t)} \\
& -\frac{\alpha \gamma}{2 Y(t) S^{\beta-2}} \frac{1}{q} \frac{d}{d s}\left(\ell^{-(\alpha \gamma)^{2} k\left(a_{i+1}-\eta\right) q \int S(t) d t} \int \ell^{(\alpha \gamma)^{2} k\left(a_{i+1}-\eta\right) q \int S(T) d T} d T\right) .
\end{aligned}
$$

Proof. Taking into (6.2.9), (6.2.10) and (6.2.3), we obtain our expected utility function, thus

$$
\begin{aligned}
h(t, s, z)= & \frac{-1}{q}\left[e^{r(T-t)}(\ln z+m(t, s))\right] \\
& +e^{-r t}\left[\int e^{r t} \frac{\left[2 k\left(\theta_{i}-r_{d}\right) C_{i+1}+\alpha \gamma u_{L}\left(a_{i+1}-\eta\right)\right]}{2 k} d t\right.
\end{aligned}
$$




$$
\left.-\int e^{r T} \frac{\left[2 k\left(\theta_{i}-r_{d}\right) C_{i+1}+\alpha \gamma u_{L}\left(a_{i+1}-\eta\right)\right]}{2 k} d T\right] .
$$

But,

$$
m(t, s)=f(t, v)=\widetilde{A}(t)+\widetilde{B}(t) v ; \quad v=s^{-2 \beta}=1,
$$

since $\beta=0$, satisfied by the assumption of case 1 and by combining, (6.2.24), (6.2.25) and (6.2.27), we have

$$
\begin{aligned}
m(t, s)= & \int w(T) d T-\int w(t) d t+e^{-\frac{(\alpha \gamma)^{2} k\left(a_{i+1}-\eta\right)}{2} q \int s(t) d t} \\
& \cdot \frac{u_{L}^{2}}{2 k^{2}}\left[\int e^{\frac{(\alpha \gamma)^{2} k\left(a_{i+1}-\eta\right)}{2} q \int s(t) d t} d t-\int e^{\frac{(\alpha \gamma)^{2} k\left(a_{i+1}-\eta\right)}{2} q \int s(T) d T} d T\right]
\end{aligned}
$$

Now, returning (6.2.28) to (6.2.26) yields

$$
\begin{aligned}
h(t, s, z)=\frac{-1}{q}\left[e ^ { r ( T - t ) } \left(\ln z+\int w(T) d T-\int w(t) d t+e^{-\frac{(\alpha \gamma)^{2} k\left(a_{i+1}-\eta\right)}{2} q \int s(t) d t}\right.\right. & \\
& \left.\left.\cdot \frac{u_{L}^{2}}{2 k^{2}}\left[\int e^{\frac{(\alpha \gamma)^{2} k\left(a_{i+1}-\eta\right)}{2} q \int s(t) d t} d t-\int e^{\frac{(\alpha \gamma)^{2} k\left(a_{i+1}-\eta\right)}{2} q \int s(T) d T} d T\right]\right)\right] \\
& +e^{-r t}\left[\int e^{r t \frac{\left[2 k\left(\theta_{i}-r_{d}\right) C_{i+1}+\alpha \gamma u_{L}\left(a_{i+1}-\eta\right)\right]}{2 k} d t}\right. \\
& \left.-\int e^{r t} \frac{\left[2 k\left(\theta_{i}-r_{d}\right) C_{i+1}+\alpha \gamma u_{L}\left(a_{i+1}-\eta\right)\right]}{2 k} d T\right] .
\end{aligned}
$$

In order to obtain the optimal strategy in (5.5), we shall firstly, obtain $h_{s}$ and $h_{z}$, and this yields

$$
\begin{aligned}
u_{s}^{*}= & \frac{\alpha \gamma\left(a_{i+1}-\eta\right)}{2 Y(t) k S^{\beta}}+(\mu-r) \ell^{r(T-t)}-\frac{\alpha \gamma}{2 Y(t) S^{\beta-2}} \\
& \cdot \frac{1}{q} \frac{d}{d s}\left(\ell^{-(\alpha \gamma)^{2} k\left(a_{i+1}-\eta\right) q \int S(t) d t} \int \ell^{(\alpha \gamma)^{2} k\left(a_{i+1}-\eta\right) q \int S(T) d T} d T\right),
\end{aligned}
$$

where, $q>0$. 
Equivalently,

$$
\begin{aligned}
u_{s}^{*}= & \frac{\alpha \gamma\left(a_{i+1}-\eta\right)}{2 Y(t) k S^{\beta}}+u_{L} \cdot \ell^{r(T-t)}-\frac{\alpha \gamma}{2 Y(t) S^{\beta-2}} \\
& \cdot \frac{1}{q} \frac{d}{d s}\left(\ell^{-(\alpha \gamma)^{2} k\left(a_{i+1}-\eta\right) q \int S(t) d t} \int \ell^{(\alpha \gamma)^{2} k\left(a_{i+1}-\eta\right) q \int S(T) d T} d T\right),
\end{aligned}
$$

where $q>0, \mu=u_{L}+r$.

\section{Sensitivity Analysis}

From (6.2.29) and (6.2.31), if we set $\alpha \gamma=0$, that is, saying that stock and time have orthogonal relationship, then the satisfaction of the contributors is reduced to (7.1) below, and the optimal investment made in stock will reduce to the product of instantaneous stock returns and the instantaneous continuous technical rate, we have, respectively

$$
\begin{aligned}
h(t, s, z)= & \frac{-1}{q}\left[e^{r(T-t)}\left(\ln z+\int w(T) d T-\int w(t) d t+\frac{u_{L}^{2}}{2 k^{2}}\left[\int d t-\int d T\right]\right)\right] \\
& +e^{-r t}\left[\int e^{r t} \frac{\left[2 k\left(\theta_{i}-r_{d}\right) C_{i+1}\right]}{2 k} d t-\int e^{r T} \frac{\left[2 k\left(\theta_{i}-r_{d}\right) C_{i+1}\right]}{2 k} d T\right]
\end{aligned}
$$

and,

$$
u_{s}^{*}=u_{L} \ell^{r(T-t)}
$$

Again, suppose, no money is paid to the next-of-kin of the dead contributors, that is, setting $a_{i+1}-\eta=0$, and this yields, respectively

$$
\begin{aligned}
h(t, s, z)= & \frac{-1}{q}\left[e^{r(T-t)}\left(\ln z+\int w(T) d T-\int w(t) d t+\frac{u_{L}^{2}}{2 k^{2}}\left[\int d t-\int d T\right]\right)\right] \\
& +e^{-r t}\left[\int e^{r t} \frac{\left[2 k\left(\theta_{i}-r_{d}\right) C_{i+1}\right]}{2 k} d t-\int e^{r T} \frac{\left[2 k\left(\theta_{i}-r_{d}\right) C_{i+1}\right]}{2 k} d T\right]
\end{aligned}
$$

and,

$$
u_{s}^{*}=u_{L} \cdot \ell^{r(T-t)}-\frac{\alpha \gamma}{2 Y(t) S^{\beta-2}} \cdot \frac{1}{q} \frac{d}{d s}\left(\int d T\right)
$$


Equivalently,

$$
u_{s}^{*}=u_{L} \cdot \ell^{r(T-t)}-\frac{\alpha \gamma}{2 Y(t) S^{\beta-2}} \cdot \frac{1}{q} F^{\prime}(s)
$$

Then, from (7.1) and (7.3), we see that when stock and time have orthogonal relationship and when no money was paid to the next-of-kin of the dead contributors, we have the same level of satisfaction on the contributors. However, both have significant effect on the satisfaction of the contributors when there is orthogonal relationship between stock and time, and also when payments are made to the next-of-kin of the dead contributors. More so, there is a significant effect on the investment made in stock.

From (6.1.21) and (6.1.24), if we set $\alpha \gamma=0$, that is, saying that stock and time have orthogonal relationship, then the satisfaction of the contributors will reduce to (7.6), but has significant negative effect on the optimal investment made in stock, and we have

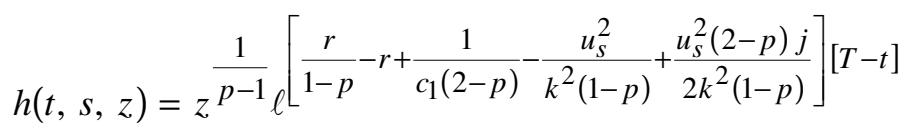

$$
\begin{aligned}
& -\ell^{-r t}\left[2 k\left(\theta_{i}-r_{d}\right) C_{i+1}\right][T-t]
\end{aligned}
$$

and,

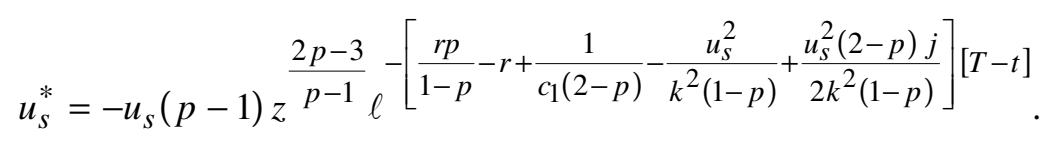

Again, suppose, no money is paid to the next-of-kin of the dead contributors, that is, setting $a_{i+1}-\eta=0$, and this yields, respectively

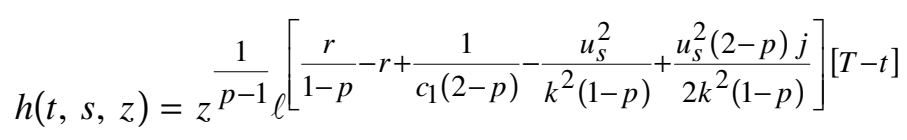

$$
\begin{aligned}
& -\ell^{-r t}\left[2 k\left(\theta_{i}-r_{d}\right) C_{i+1}\right][T-t]
\end{aligned}
$$

and

$$
u_{s}^{*}=-u_{s}(p-1) z^{\frac{2 p-3}{p-1}} \ell-\left[\frac{r p}{1-p}-r+\frac{1}{c_{1}(2-p)}-\frac{u_{s}^{2}}{k^{2}(1-p)}+\frac{u_{s}^{2}(2-p) j}{2 k^{2}(1-p)}\right][T-t] .
$$


Then, from (7.6) and (7.8), we see that when stock and time have orthogonal relationship and when no money was paid to the next-of-kin of the dead contributors, we have the same level of satisfaction on the contributors. However, both have significant effect on the satisfaction of the contributors when there is orthogonal relationship between stock and time, and also when payments are made to the next-of-kin of the dead contributors.

From (7.7) and (7.9), we observe that the introduction of the orthogonal relationship between stock and time, and the nonpayment of benefits to the next-of-kin of the dead contributors have a negative significant effect on the money invested in stock. However, both introductions have the same negative effect (i.e., a decline in stock investment) on the investment made in stock.

\section{Numerical Illustration}

A numerical example of the proposed model was given to demonstrate the dynamic behaviour of a DC pension fund and optimal investment strategy. Nigeria-National Pension Fund Administration (NNPFA) real data was used to illustrate the efficiency of the proposed model. The parameters used are summarized in Table 1 , for $T=35$. $t=0,5,10,15,20,25,30,35$ with $i=4,5, \ldots, n-1$ and $\theta_{4}=4, \theta_{5}=5, \theta_{6}=6, \ldots$, $\theta_{n}=n$.

Table 1. Parameters and their respective values.

\begin{tabular}{|l|c|c|}
\hline Name of parameter & Symbol used & Values \\
\hline Constant rate of interest & $r$ & 0.02 \\
\hline Expected stock returns & $\mu$ & 0.10 \\
\hline Instantaneous stock returns & $u_{S}=u_{L}$ & 0.07 \\
\hline Stock volatility & $k$ & 0.55 \\
\hline Risk aversion & $p=q$ & 0.50 \\
\hline Rate of contribution & $r_{d}$ & 0.075 \\
\hline Management fee & $\eta$ & 0.025 \\
\hline
\end{tabular}




\section{Results}

Figure 1 describes the investment strategy for the CRRA utility function under the constant elasticity of variance $(\mathrm{CEV})$ model using (6.1.21) when $c_{1}=\frac{1}{2}\left(a_{i+1}-\eta\right)^{2}$ $+\frac{3}{8}(\alpha \gamma)^{2}\left(a_{i+1}-\eta\right)^{2}, j=\ell^{-2 \beta}, \beta=0 \Rightarrow j=1$. It shows that the stock price has significant effect on the optimal investment made in stock.

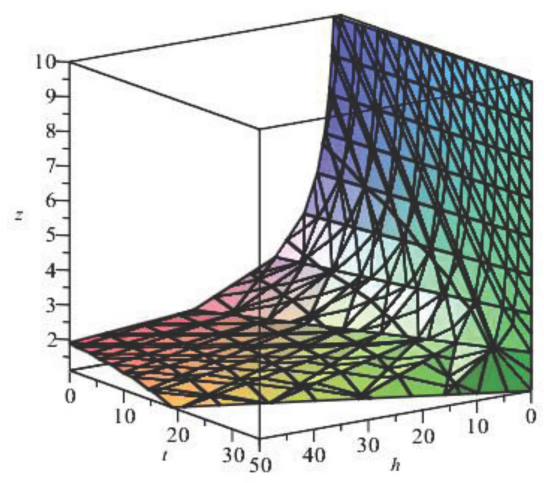

Figure 1. Investment strategy under CEV using (6.1.21).

Figure 2 shows the influence instantaneous stock returns on the optimal investment strategies using (6.1.24) with $c_{1}=\frac{1}{2}\left(a_{i+1}-\eta\right)^{2}+\frac{3}{8}(\alpha \gamma)^{2}\left(a_{i+1}-\eta\right)^{2}, Y(t)=1000$. It reveals that the optimal investment policies increase with time. That is, as the time passes on, investment in riskless asset decreases. Results suggest that the pension fund manager maintains diversifying the portfolio by investing more in stock since the optimal investment strategies in risky assets increase with time.

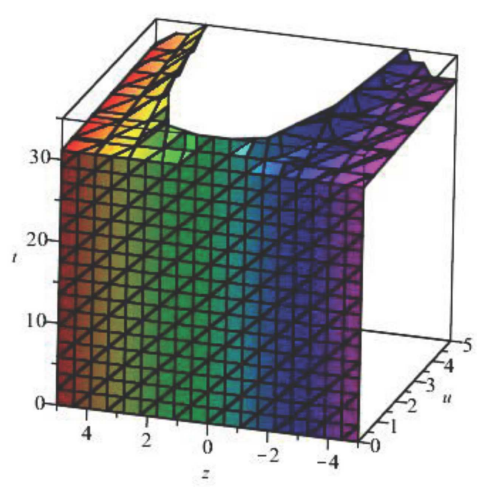

Figure 2. Influence of instantaneous stock returns on the optimal investment strategies. 
Figure 3 describes the investment strategy for the CARA utility function under the constant elasticity of variance (CEV) model using (6.2.26). It shows that increase in the interest rate volatility decreases the optimal investment strategy; this tells the pension fund manager to invest more in a riskless asset to hedge risk.

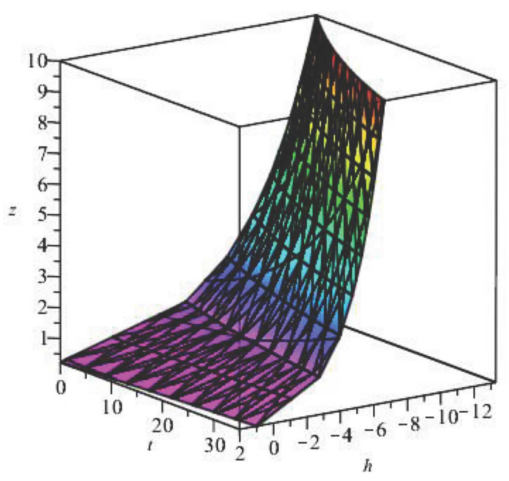

Figure 3. Investment strategy under CEV using (6.1.26).

Figure 4 presents the impact of instantaneous stock returns on the optimal investment strategies. It points out that as instantaneous stock returns increase the optimal investment strategies decreases.

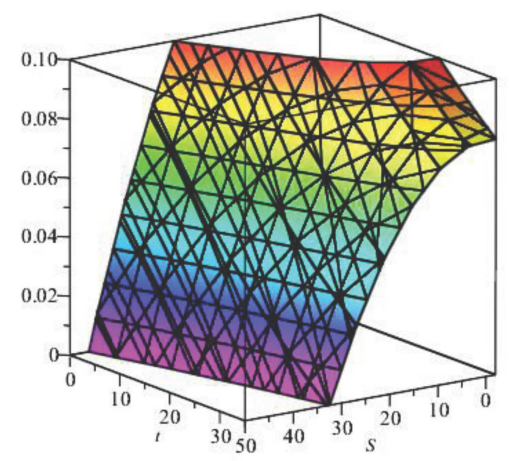

Figure 4. Influence of instantaneous stock returns on the optimal investment strategies using (6.2.30).

\section{Conclusion}

We studied and modified the optimal investment strategy for annuity contract under the constant elasticity of variance as in literature to show that the elastic parameter takes values other than unity. We also constructed the optimal pension wealth investment 
strategy during the decumulation phase, in a defined contribution (DC) pension scheme and obtained the explicit solution of the constant relative risk aversion (CRRA) and constant absolute risk aversion (CARA) utility functions.

\section{References}

[1] E. E. Akpanibah, B. O. Osu, K. N. C. Njoku and Eyo O. Akak, Optimization of wealth investment strategies for a DC pension fund with stochastic salary and extra contributions, International Journal of Partial Differential Equations and Applications 5(1) (2017), 33-41.

[2] P. Battocchio and F. Menoncin, Optimal pension management in a stochastic framework, Insurance Math. Econom. 34(1) (2004), 79-95.

[3] J.-F. Boulier, S. Huang and G. Taillard, Optimal management under stochastic interest rates: the case of a protected defined contribution pension fund, Insurance Math. Econom. 28(2) (2001), 173-189.

[4] A. J. G. Cairns, D. Blake and K. Dowd, Stochastic lifestyling: optimal dynamic asset allocation for defined contribution pension plans, J. Econom. Dynam. Control 30(5) (2006), 843-877.

[5] C. Zhang and X. Rong, Optimal investment strategies for DC pension with stochastic salary under the affine interest rate model, Discrete Dyn. Nat. Soc. 2013, Art. ID 297875, 11 pp. http://dx.doi.org/10.1155/2013/297875.

[6] J. C. Cox, J. E. Ingersoll and S. A. Ross, A theory of the term structure of interest rates, Econometrica 53(2) (1985), 385-407.

[7] G. Deelstra, M. Grasselli and P.-F. Koehl, Optimal investment strategies in the presence of a minimum guarantee, Insurance Math. Econom. 33(1) (2003), 189-207.

[8] J. Gao, Stochastic optimal control of DC pension funds, Insurance Math. Econom. 42(3) (2008), 1159-1164.

[9] J. Gao, Optimal portfolios for DC pension plans under a CEV model, Insurance Math. Econom. 44 (2009), 479-490.

[10] J. Gao, Optimal investment strategy for annuity contracts under the constant elasticity of variance (CEV) model, Insurance Math. Econom. 45(1) (2009), 9-18.

[11] M. Jonsson and R. Sircar, Optimal investment problems and volatility homogenization approximations, in: Modern Methods in Scientific Computing and Applications, vol. 75 of NATO Sci. Ser. II, pp. 255-281, Berlin, Germany: Springer, 2002. 
[12] K. N. C. Njoku, B. O. Osu, E. E. Akpanibah and R. N. Ujumadu, Effect of extra contribution on stochastic optimal investment strategies for DC pension with stochastic salary under the affine interest rate model, Journal of Mathematical Finance 7 (2017) 821-833.

[13] Nigerian Pension Reform Act of 2004

[14] P. K. Mwanakatwe, L. Song and E. Hagenimana, Management strategies for a defined contribution pension fund under the Hull-White interest rate model, Advances in Intelligent Systems Research (AMMS) 153 (2017), 239-244.

[15] L. Wang and Z. Chen, Nash equilibrium strategy for a DC pension plan with statedependent risk aversion: a multiperiod mean-variance framework, Discrete Dyn. Nat. Soc. 2018, Art. ID 7581231, 17 pp. https://doi.org/10.1155/2018/7581231.

[16] J. Xiao, Z. Hong and C. Qin, The constant elasticity of variance (CEV) model and the Legendre transform-dual solution for annuity contracts, Insurance Math. Econom. 40(2) (2007), 302-310. 